Décadrages Décadrages

cınéma, à travers champs Cinéma, à travers champs

$11 \mid 2007$

Terrence Malick

\title{
Entre distance et immersion : des voix qui glissent sur le monde
}

Les locuteurs over dans les films de Terrence Malick

\section{Alain Boillat}

\section{OpenEdition}

\section{Journals}

Édition électronique

URL : http://journals.openedition.org/decadrages/393

DOI : $10.4000 /$ decadrages.393

ISSN : 2297-5977

Éditeur

Association Décadrages

Édition imprimée

Date de publication : 1 octobre 2007

Pagination : $9-20$

ISBN : 978-2-9700582-6-7

ISSN : 2235-7823

Référence électronique

Alain Boillat, « Entre distance et immersion : des voix qui glissent sur le monde », Décadrages [En ligne], 11 | 2007, mis en ligne le 01 octobre 2009, consulté le 19 avril 2019. URL : http:// journals.openedition.org/decadrages/393 ; DOI : 10.4000/decadrages.393 


\section{Entre distance et immersion : des voix}

\section{qui glissent sur le monde}

\section{Les locuteurs over dans les films}

\author{
de Terrence Malick
}

par Alain Boillat

Rares sont les cinéastes qui, au sein de la production hollywoodienne, recourent à la voix-over $\mathbf{1}$ avec la constance et l'inspiration dont témoignent les films de Terrence Malick. Tous ses longs métrages sont en effet parcourus par ce type d'occurrence vocale qui détermine grandement l'impact esthétique et perceptif qu'ils produisent sur un (audio)spectateur happé par leur envoûtante "musicalité", invité à une sollicitation des sens plutôt qu'à une fixation du sens. La voix-over plane par intermittence au-dessus des actions, s'associant au bercement du montage pour produire un effet diffus de suspension. Elle est donc loin d'être réduite à un "procédé» exclusivement soumis à des impératifs narratifs comme l'explication du contexte historique - dans The Thin Red Line (La Ligne rouge, 1998), les informants verbaux ne nous apprennent par exemple rien d'autre sur la bataille de Guadalcanal que les réflexions très générales qu'elle suscitent chez les protagonistes - ou la psychologisation des personnages (en raison d'une tendance à situer les commentaires over à un niveau supra-individuel). Le texte over et le ton à la fois détaché et méditatif sur lequel il est prononcé participe du régime contemplatif qui prédomine dans les œuvres de Malick. Ses films ne sont en effet pas exempts d'une esthétisation qui serait outrancière si la voix-over ne problématisait, parfois à l'insu des personnages-narrateurs, le rapport des individus au monde et la dimension stéréotypée de la représentation. Ce questionnement sur l'exhibition du "cliché» - à prendre littéralement si l'on pense à la présence d'images fixes dans les deux premiers films de Malick - permet à Badlands (La Balade sauvage, 1973), dont l'action est située au début des années 1960, d'échapper en partie à cette nostalgie escapiste qui caractérise pour Jameson le «post-modernisme» des films
1 A l'instar de Jean Châteauvert (Des mots à l'image. La Voix over au cinéma, Nuit Blanche/ Méridiens Klincksieck, Québec/Paris, 1996), j'utiliserai le terme "over " pour qualifier une voix qui ne peut être entendue par les personnages appartenant au monde du film, réservant l'expression "voix off" à des cas où le locuteur, diégétique, se trouve hors-champ ("off screen"). 
2 Fredric Jameson, Postmodernism, or the Cultural Logic of Late Capitalism, Duke University, Durnham, 1999.

3 Elisabeth Bronten, "Recycling von Gewalt und Gesetzlosigkeit", dans Hans Helmut Prinzler et Gabriele Jatho (éd.), New Hollywood 1967-1976. Trouble in Wonderland, Bertz, Berlin, 2004, pp. 15-32.

4 Stanley Cavell, La Projection du monde, Belin, Paris, 1999, p. 258.

5 Dans la typologie genettienne, cet adjectif qualifie des narrateurs qui sont par ailleurs présents en tant que personnages dans l'histoire qu'ils racontent (Gérard Genette, Figures III, Seuil, Paris, 1972, p. 252). La distinction homodiégétique vs. hétérodiégétique est particulièrement importante au cinéma dans la mesure où une même voix est, dans le premier cas qui s'applique à tous les films de Malick, alternativement entendue over et in (lorsque l'acteur qui incarne le personnage-narrateur est montré parlant à l'image). La reconnaissance du timbre de la voix-over engendre une certaine incarnation des locuteurs over et un ancrage dans la diégèse de la subjectivité des textes qu'ils profèrent (voir à ce propos François Jost, Un monde à notre image, Méridiens Klincksieck, Paris, par exemple p. 99). Toutefois, l'assignation d'un visage à une voix-over est passablement enrayée chez Malick par la mise en retrait de la voix in qui s'opère soit par suppression (personnages taciturnes, dialogues inaudibles), soit par "déliaison" (le statut de la voix demeurant ainsi indécidable, situé qu'il est entre le off et le over).

6 Sarah Kozloff, Invisible Storytellers, Voiceover Narration in American Fiction Film, University of California Press, Berkeley/Los Angeles/ London, 1988, pp. 116-117.

7 II en est question dans l'un des rares entretiens accordés par Malick à une revue spécialisée: "-Pensiez-vous dès l'origine au contrepoint entre le commentaire off de Holly et les images? - Absolument, mais j'ai essayé de le minimiser dans le scénario car les grandes compagnies distributrices ont tendance à craindre le commentaire off - ils trouvent cela peu cinématographique. [...] Lorsque le texte n'a pas un rapport direct avec l'action, comme dans Badlands, cela me semble mieux fonctionner." (Michel Ciment, "Entretien avec Terrence Malick", Positif, no 170, juin 1975, p. 31). du New Hollywood comme American Graffiti (George Lucas, 1973) ou Sugarland Express (Steven Spielberg, 1974) 2 ${ }^{2}$ Elisabeth Bronten parle à propos de Malick de "nostalgie ironique", une notion qui convient bien pour qualifier les enjeux de la prise de distance instaurée par l'énonciation filmique relativement au discours des personnages ${ }^{3}$. Rappelons à cet égard que Stanley Cavell dit de la jeune fille interprétée par Sissy Spacek dans Badlands qu'elle confie "la narration de sa vie au présentoir de formules prises au hasard sur les rayons des magazines» $\mathbf{4}$.

La singularité «rythmique» de la voix-over chez Malick me semble s'être affirmée dans ses deux derniers films, peut-être en raison de la forte présence du motif aquatique auquel s'accordent, sur le plan formel, les épanchements incantatoires de la voix-over et le flux des plans, auxiliaires d'une immersion du spectateur désormais dépourvue des effets de distanciation (au sens brechtien du terme) à l'œuvre dans ses deux premières productions. En effet, le caractère hypnotique de ces voix dont la mélodie s'accorde aux traversées lancinantes des terres inconnues de The Thin Red Line (La Ligne rouge, 1998) ou de The New World (Le Nouveau Monde, 2005) enjoint au contraire le spectateur à se laisser happer par le monde décrit, tandis que, dans les deux films précédents du cinéaste, Badlands et Days of Heaven (Les Moissons du ciel, 1978), l'intérêt des voix-over homodiégétiques 5 rétrospectives réside bien plus dans leurs implications énonciatives. Leur spécificité tient au fait que la structure de ces films diffère des pratiques dominantes du cinéma «classique» en ce qu'elle repose, ainsi que l'a montré Sarah Kozloff dans un chapitre consacré à l'ironie, sur des «narrateurs non fiables» ("unreliable narrators") 6 . Le récit de Badlands est placé sous la direction d'une adolescente naïve, alors que Les Moissons $d u$ ciel nous est conté par une fillette de douze ans. Comme cette dernière représente un personnage périphérique, j'écarterai ce film pour me concentrer sur Badlands, où la narratrice Holly est une figure centrale: fréquents, ses commentaires over pèsent fortement sur l'appréhension que nous avons du film.

\section{Badlands: l'indifférente nature (du sujet parlant)}

Dans Badlands, le détachement extrême que la narratrice affecte envers les agressions gratuites perpétrées par son ami de l'époque, Kit (Martin Sheen), domine le récit, le spectateur devant comprendre «entre les lignes» que le film n'encourage pas l'adhésion à ses propos, et dans les interstices qui séparent le référent verbal de celui des images que l'idylle du couple dépeinte over ne correspond pas à la situation vécue dans la diégèse. De ce fait, le film souscrit à un principe souvent qualifié de contrepoint $\mathbf{7}$, technique traditionnellement prônée parce qu'elle instaure- 
rait un rempart à une prétendue «redondance» de la parole et de l'image, redondance qui constitue une véritable bête noire des théoriciens du cinéma ${ }^{8}$. La distance résultant du caractère inapproprié des remarques de la narratrice de Badlands enjoint le spectateur à contester la position faîtière communément attribuée à la voix-over ${ }^{9}$, et à inclure cette dernière dans une stratification énonciative plus vaste où une instance de niveau supérieur "commente» les dires de Holly à travers leur énonciation même10. Par ailleurs, les expressions utilisées par la narratrice over révèlent l'influence de Kit, les commentaires de ce dernier n'étant pas toujours rapportés en style indirect, mais parfois totalement intégrés au discours de Holly. La "voix-je», comme l'appelle Michel Chion ${ }^{\mathbf{1 1}}$, est donc condamnée à la citation (comme Kit imite par son attitude James Dean), privée de l'expression personnelle qui la caractérise. Aussi l'attribution de la responsabilité énonciative des propos tenus over est-elle fluctuante, voire incertaine.

Bien que le traitement de la voix-over dans Badlands présente de nombreuses similitudes avec ce que l'on trouve couramment dans le cinéma hollywoodien $\mathbf{1 2}$, il annonce par certains aspects des pistes que Malick approfondira dans ses films ultérieurs. Ainsi, en dépit de l'importance qualitative des moments de parole, les conversations entre les personnages sont rares et, lorsqu'elles ont lieu, s'avèrent extrêmement superficielles. Les silences du dialogue, qui permettent souvent à des vues de la nature de s'immiscer dans le récit de la vie du couple en cavale, contrastent avec l'application un peu puérile dont la narratrice fait preuve en nous contant ses aventures. Lorsqu'il rencontre Holly, Kit lui déclare ne pas être gêné par son laconisme; artificiellement rebelle, il donne toujours l'impression d'avoir beaucoup à dire, mais lorsqu'il s'empare d'un dictaphone pour enregistrer ses propres paroles, on constate que sa verve à proclamer de grands idéaux s'épuise rapidement pour retomber dans l'horizon étroit de leur quotidien de fugitifs. La communication s'instaure avant tout entre le film et son spectateur par l'intermédiaire de la voix-over, tandis que les situations décrites sont marquées du sceau de la vacuité ou de l'incommunicabilité. La présence d'une unique voixover détachée des événements, en déshérence de corps agissant, renforce cette impression que les personnages sont fondamentalement étrangers à eux-mêmes. Enfin, la séquence du Stéréopticon (dispositif optique à l'aide duquel Holly contemple des images surannées qu'elle associe au passé de sa famille) occasionne une rupture dans le référent temporel de la voix-over qui, dans le reste du film, raconte invariablement au passé. En effet, dans ce passage, le texte over prend momentanément ancrage, après un détour par le conditionnel passé, dans le présent de
8 Concernant la valorisation du contrepoint son/ image, voir par exemple Siegfried Kracauer, Theory of film, The Redemption of Physical Reality, Oxford University Press, Londres, 1960, pp. 117-119. Par la suite, Malick n'évite à mon sens plus cette redondance, mais il substitue au contrepoint un autre modèle, moins axé sur la dimension sémantique: celui de la "correspondance" entre les matières de l'expression cinématographique.

9 La façon dont la "voix narrative" a été conceptualisée dans le champ des études littéraires et dans la théorie du cinéma est en elle-même révélatrice de cette tendance à unifier l'origine d'un discours (pourtant pluristratifié) autour d'une instance anthropocentrique et surplombante. Pour une discussion et une critique de ce modèle, voir le chapitre 6 de mon ouvrage Du bonimenteur à la voix-over. Voix-attraction et voix-narration au cinéma, Antipodes, Lausanne, 2007.

10 On peut rendre compte de ce type d'énonciation à l'aide de théories linguistiques, notamment en recourant au modèle polyphonique proposé par Oswald Ducrot (Le Dire et le dit, Minuit, Paris, 1984, chapitre 8, "Esquisse d'une théorie polyphonique de l'énonciation", pp. 171-233).

11 Michel Chion, La Voix au cinéma, Editions des Cahiers du cinéma, Paris, 1982, chapitre 3.

12 Malick déclare par exemple que "cela vous permet de résoudre rapidement des problèmes que les spectateurs d'aujourd'hui ne prennent aucun intérêt à vous voir résoudre: comment ils sont arrivés là, le passage du temps, etc." ("Entretien avec Terrence Malick», op. cit., p. 31). 
13 Jean Châteauvert précise à ce propos: "Sur le plan de l'analyse, le monologue intérieur se distingue de la 'narration en voix-over, en ce qu'il se conjugue généralement au présent et révèle les réflexions, angoisses, doutes, etc., qu'éprouve un personnage dans une situation" (Des mots à l'image..., op. cit., p. 143). Or Malick se plaît à brouiller constamment ces distinctions en associant le présent et l'extraction du personnage de la situation vécue, ou inversement en usant du passé tout en nous plongeant dans des pensées contemporaines des événements visualisés.

14 Christa M. Haeseli, " 'The world was like a faraway planet to which I could never return.' Die Subjektkonzeption der Voice-Over in Terrence Malicks Badlands", Montage/av, Figuren und Perspektive (1), 15.2.2006, pp. 115-134.

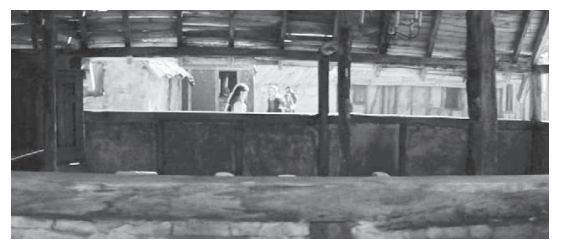

1

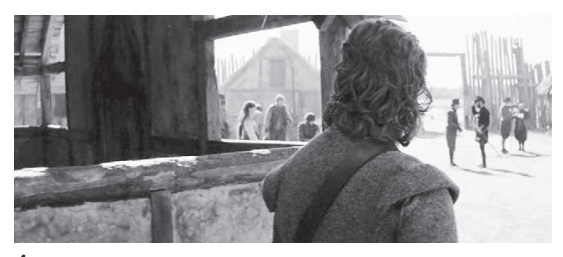

4 l'observation des vues, qui apparaissent simultanément au spectateur en plein écran. Un tel changement de temps verbal provoque une indécision qui affecte autant notre compréhension du temps - par ailleurs perturbée par un recours à l'itératif sur des portions de film inhabituellement longues - que la façon dont nous appréhendons la voix-over, qui tend parfois au monologue intérieur sans obéir totalement à ce régime ${ }^{\mathbf{1 3}}$.

En tant que scénariste, Malick use constamment de tels effets de décalage: les paroles over ne correspondent pas aux événements dont elles rendent compte, et les personnages, foncièrement esseulés en dépit de la romance, "flottent» sur leurs aventures plus qu'ils ne les vivent. Comme l'a expliqué récemment Christa Haeseli, Badlands se démarque d'une conception du sujet de type cartésien, basée sur la raison et l'existence d'une entité stable: le «je» de la narratrice est au contraire une instance totalement vide $\mathbf{1 4}$. Aucun film de Malick ne recourant au principe du récit-cadre visualisé, la désincarnation des narrateurs renforce l'impression d'une absence d'emprise sur un monde qui demeure fondamentalement extérieur, inaccessible à ceux qui tentent d'en rendre compte par des mots. En introduisant des sociétés "primitives" comme les tribus polynésiennes et les Algonquins d'Amérique, La Ligne rouge et Le Nouveau Monde laisseront par contre entrevoir la possibilité d'une harmonie entre l'homme et la nature. Certes, l'individuum n'existe pas dans le panthéisme malickien, mais la parole peut servir à mettre en relation les diverses parties du tout. En outre, à partir de son troisième long métrage, Terrence Malick ne se limite plus à une voix-over unique, mais en introduit plusieurs. Nous verrons comment s'organisent ces narrations chorales.

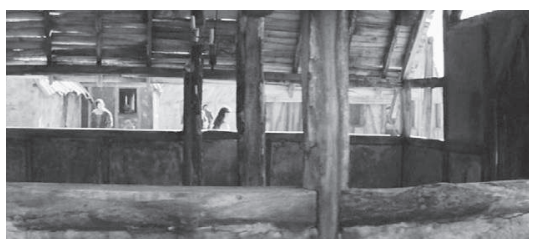

2

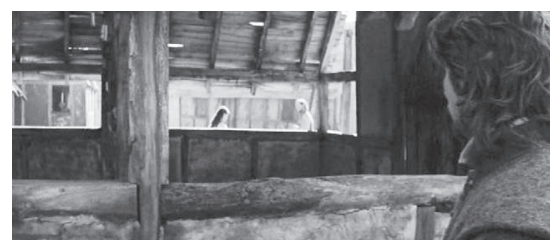

3

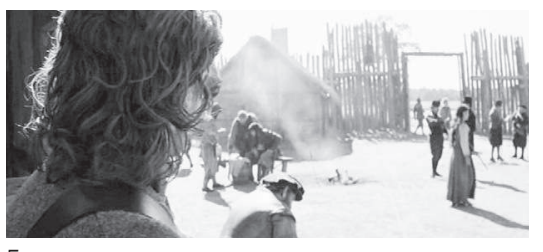

5

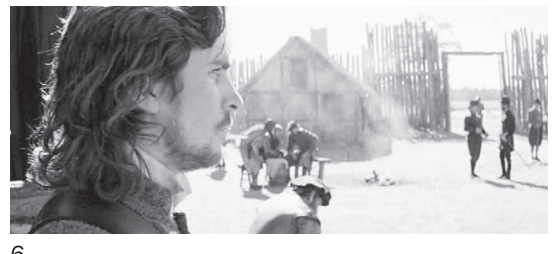

6 


\section{Sinuosités des récits polyphoniques}

Dans Le Nouveau Monde, la première partie (à laquelle nous consacrerons la fin du présent article) est prise en charge par les voix-over de l'officier John Smith (Colin Farrell) et de la plus jeune fille du roi indigène, Pocahontas (Q'Orianka Kilcher). Dans le dernier tiers du film, un changement intervient: alors que la civilisation est en marche, Pocahontas, abandonnée par son amant qui lui préfère l'aventure, erre hagarde et muette parmi les colons et les charpentes d'une ville en construction. Alors qu'elle donne à boire à un prisonnier mis aux fers, un insert nous montre un personnage pensif, le regard perdu au loin. John Rolfe (Christian Bale) entre ainsi dans le film, et dans la vie de l'héroïne. On le découvre tout d'abord en situation d'observation, ce qui permet à Malick de nous faire épouser progressivement son regard. Sur le plan visuel, cet ancrage s'opère notamment au gré d'un travelling latéral qui suit la jeune femme aperçue à travers un baraquement ajouré - obstruction partielle de la vue qui souligne l'importance du cadre et du «point de vue»15 -, puis s'immobilise à la hauteur de John apparu au premier plan, enfin calque son mouvement sur le déplacement de ce dernier qui continue de fixer Pocahontas désormais sortie du champ (fig. 1-6). On la retrouve dans le plan suivant, présentée comme l'objet du regard de John, alors qu'elle verse dans un récipient l'eau qu'elle a puisée. Lorsque John réapparaît à l'avant-plan, sa voix-over retentit, joignant la focalisation à l'ocularisation dans une phrase qui thématise le rapport du sujet percevant à son objet: "When I first saw her...»(fig. 7). Cette seconde voix-over masculine qui ne se manifeste qu'après une durée correspondant à ce qui pourrait être la fin possible d'un long métrage (90 minutes) peut surprendre, puisque le film avait habitué le spectateur au rythme des duos de Pocahontas et Smith. Contrastant avec les monologues lyriques chuchotés par Colin Farrell, la voix de Bale, claire et posée, signale en fait une bifurcation du récit, fraie une nouvelle piste dans le parcours de l'héroïne. Les deux John (Smith et Rolfe) représentent chacun une facette de la conquête des territoires amérindiens que l'on trouve dans de nombreux westerns: la perpétuelle fuite en avant du pionnier ou l'exploitation du sol menée par le bâtisseur sédentaire. La courbe dramatique du Nouveau Monde se noue ainsi autour de ce relais d'une voix-over par une autre, accordant à ce procédé une fonction structurelle primordiale.

Dans La Ligne rouge, les énonciateurs over ne s'excluent pas mutuellement, mais s'expriment en alternance au sein d'une composition chorale plus disparate. Pour rendre compte de ces prises de paroles spontanées et presque aléatoires qui ponctuent ce film, Michel Chion parle de
15 Pour reprendre la terminologie de François Jost (L'CEil-caméra, PUL, Lyon, 1987), on passe dans cette séquence d'une ocularisation zéro (qui s'accompagne d'un marquage énonciatif, le mouvement d'appareil affichant la présence de la caméra) à une ocularisation interne primaire (personnage en amorce) puis secondaire (le champ/contrechamp atteste qu'il l'observe, alors que le statut du premier insert de John demeure ambigu, l'identité de l'espace occupé par les deux personnages n'ayant pas encore été établie).

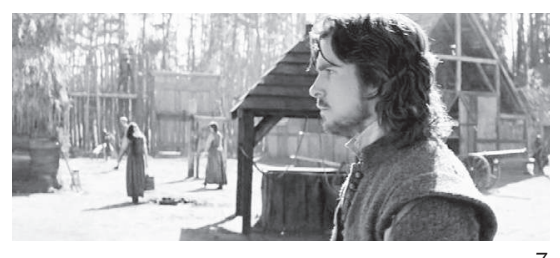


16 Michel Chion, La Ligne rouge, Editions de la Transparence, Chatou, 2005, p. 59.

17 Ces sept voix sont celles des acteurs suivants: Jim Caveziel, Nick Nolte, Ben Chaplin, Dash Mihok, Adrien Brody, Sean Penn et Miranda Otto. Cette dernière interprète l'épouse de Bell et constitue l'unique personnage féminin à bénéficier d'une voix-over (la mère de Witt demeure muette). Motivée par le contexte épistolaire, cette voix n'apparaît qu'à la fin du film: Bell lit la lettre de sa femme que nous avons vue de façon récurrente dans des inserts muets (flash-backs qui, imperceptiblement, en viennent à représenter la simultanéité d'une attente griffithienne). Malick aurait pu choisir la voix de l'acteur qui incarne le soldat (Ben Chaplin), d'autant que nous l'entendons ailleurs en régime over. Le surgissement de la voix de femme vient toutefois souligner l'altérité de celle-ci, qui demande le divorce à celui qui s'est sans cesse raccroché à l'idée de la retrouver. La désillusion est renforcée par une manifestation vocale qui vient individualiser tardivement ce personnage tout en le congédiant du film. Notons que la voix de Miranda Otto (ou celle d'une autre actrice qui l'aurait doublée) retentit durant l'un des souvenirs de Bell ("Viens, viens me rejoindre ", dit-elle en s'avançant dans la mer): bien que l'actrice soit visible à l'image, ses lèvres demeurent immobiles, comme si la voix provenait d'outre-tombe.

18 II est révélateur que Michel Chion souligne en préambule du découpage qu'il propose dans l'annexe de son ouvrage sur le film la difficulté que rencontre le spectateur, "même après plusieurs visions du film [...], [à] attribuer certaines voix à leur propriétaire" (M. Chion, La Ligne rouge, op. cit., p. 85); dans le descriptif de la séquence 34 , il se contente d'ailleurs d'indiquer "une voix médite (voix intérieure)" (id., p. 95 ; je souligne). «petits poèmes naïfs, [d']îlots de mots avec l'espace autour»16. C'est que chaque occurrence constitue une respiration singulière et autonome qui participe de la solitude des êtres face à des situations extrêmes. Le principe du relais ne s'applique qu'à une seule des sept voix-over reconnaissables du film 17, celle de Welsh (Sean Penn), qui ne commence à se faire entendre qu'après la mort de Witt (Jim Caviezel), principal narrateur du film (si tant est qu'une hiérarchie puisse être établie). La séquence-pivot qui connaît le surgissement de cette nouvelle voix nous montre Welsh se recueillant devant la tombe sommairement créée pour Witt: l'adresse à un mort ("tu es dans une boîte») semble elle-même affectée de la désincarnation de l'interlocuteur muet, et de la déliaison propre aux prières du film.

Bien que La Ligne rouge appartienne à un genre, le film de guerre, qui peut susciter certaines attentes relatives à l'inscription du récit dans le contexte historique de référence (la guerre du Pacifique), Malick propose un texte over qui se situe aux antipodes du factuel, comme l'annonce la phrase inaugurale dans laquelle le soldat Witt synthétise sous une forme interrogative l'oxymore résultant de l'alliance des deux grands motifs du film : "Pourquoi cette guerre au milieu de la nature?» Malick, plutôt que de privilégier la transmission d'un savoir éprouvé, s'interroge avec le public sur le spectacle de la nature qu'il donne à voir dans sa grandeur et son mystère. La plupart du temps, les pensées se manifestent over dans des moments de torpeur, un sentiment qui envahit tous les soldats (et la plupart de leurs supérieurs), plongés dans une absence qui les isole les uns des autres dans l'histoire, mais les lie sur le plan formel. Il est d'ailleurs souvent malaisé d'identifier dans ce film les changements de narrateurs $\mathbf{1 8}$, comme si toutes les voix fusionnaient dans un questionnement commun sur la condition humaine et sur les rapports de l'homme à une nature supérieure et indifférente. Lorsque Bob Witt s'avance parmi les premiers blessés américains découverts sur l'île, il se demande over si tous les hommes n'auraient pas une âme commune identique ("one big soul, where everybody is a part of»), si tous les visages n'appartiendraient pas à un même homme ("all faces of the same man, one big self») : le film donne l'impression qu'il en va ainsi des voix, tant l'ancrage dans des personnages agissants (parfois non nommés) est discret ou ambigu. Il peut sembler paradoxal d'utiliser la voix pour briser l'individualisation, alors qu'il s'agit justement là de quelque chose qui est propre à chacun. Le ton méditatif et intime des voix-over se démarque toutefois fortement de celui des répliques prononcées in par les mêmes acteurs, qui en vociférant, qui dans le brouhaha des cris et des tirs. A l'instar des invocations du Nouveau Monde, seules les prières de Staros 
(Elias Koteas) semblent simultanément ressortir aux régimes in et over. Il arrive néanmoins qu'un personnage censé parler over soit visualisé simultanément dans des plans où ses lèvres ne se meuvent pas; une telle situation de "déliaison" entre la voix et l'image provoque un effet d'étrangeté.

A l'exception du premier monologue intérieur du lieutenant-colonel Tall (Nick Nolte), qui motive et annonce son obstination future à vouloir attaquer frontalement la crête au mépris des pertes humaines, le texte over contribue assez peu à construire les personnages, si ce n'est dans leur rapport "philosophique» au monde, et plus précisément dans la façon dont ils négocient l'omniprésence de la mort, cette "frontière » à laquelle le titre du film fait référence, cet au-delà suggéré par la matérialité sonore de voix-over foncièrement fantomatiques 19. Lorsque les mots dont usent les narrateurs instaurent une coréférence avec la représentation iconique, ils introduisent le plus souvent une dimension universelle qui décontextualise le propos et neutralise tout rapport de cause à effet entre les actes et les commentaires. Une exclamation obsessionnellement répétée échappe à cette règle : le soldat Doll (Dash Mihok) s'écrie "J'ai tué un homme» lors de son premier meurtre. En raison de la rareté d'une référence à une action qui vient de se produire, l'exclamation de Doll est mise en exergue, et par là les implications éthiques et psychologiques de son acte. Par ailleurs, cette phrase se singularise également des autres énoncés over qui, eux, sont dominés par le temps verbal du présent dans un usage plus gnomique que déictique, la généralité des propos impliquant une intemporalité qui neutralise le renvoi à la situation temporelle de l'énonciation. En utilisant un temps passé («I killed...»), le soldat souligne le caractère irrémédiable de son geste funeste; le statut over de cette phrase suggère le caractère révolu de l'événement, qui déjà quitte l'univers matériel pour résonner dans l'intériorité du personnage confronté à la mort.

Dans La Ligne rouge, la focalisation interne n'est pas multiple, mais variable 20: la polyphonie qui en découle (ou qui la fonde) ne s'inscrit donc pas dans un jeu tapageur sur le récit tel qu'en pratiquent des cinéastes du type de Tarantino (ou, pour prendre un sujet proche, Eastwood dans son récent diptyque), mais au contraire tend à suspendre le narratif au profit de touches "impressionnistes » qui émaillent la matière sonore du film. L'éviction d'informants narratifs renforce ainsi l'éclatement polyphonique des individualités dont résulte chez Malick un mode d'intériorisation fort différent des conventions anthropocentriques et psychologisantes qui régissent habituellement la représentation audiovisuelle. Car si tous ses personnages sont indubitablement absorbés, ils
19 L'une des voix du film surgit littéralement d'outre-tombe: celle du Japonais agonisant auquel s'adresse Dale (Arie Verveen), que ce dernier entend à nouveau plus tard, Iorsqu'il se repent d'avoir dépouillé les cadavres. Or il s'agit là d'une voix qui nous apparaît véritablement en tant que matière vocale, puisque le film ne sous-titre aucune réplique des Japonais.

20 La focalisation interne est dite "multiple" lorsque le même événement est raconté par des personnages différents (voir G. Genette, op. cit., p. 207). 
21 On retrouvera ce motif dans un segment en focalisation interne sur Smith, lorsque ce dernier décrit le mode de vie dans le camp des autochtones; l'insert sur un plan d'eau brouillé par des éclats de gouttes fait précisément suite au mot "rêve" ("Ça existe, ce que je croyais être un rêve"). ne le sont parfois pas uniquement par leurs pensées, mais aussi par le monde qui les entoure et fait l'objet des réflexions verbalisées over. Son dernier film portera à son paroxysme cet éclatement de la figuration vocale et visuelle de l'individu.

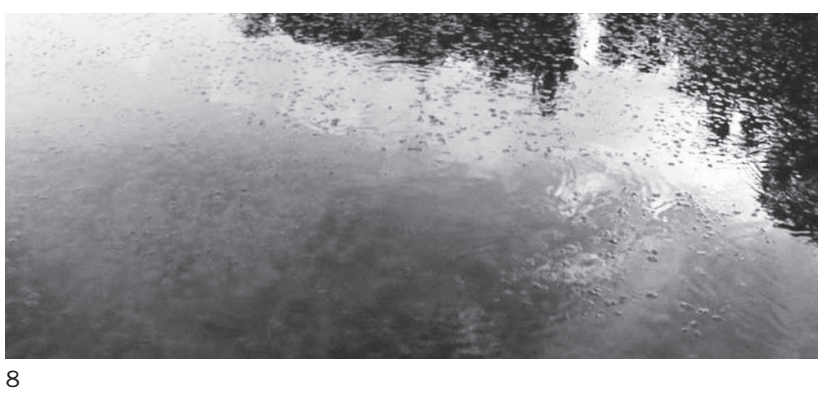

\section{Plan d'eau, voix du ciel: l'entrée dans le Nouveau Monde}

Le dernier opus malickien s'ouvre sur deux plans de pré-générique qui, telle une épigraphe, donnent littéralement l'esprit du film: "Viens esprit, aide-nous à chanter l'histoire de notre terre», chuchote une voix féminine qui n'a pas encore été associée au personnage de Pocahontas. La prière ne s'adresse pas au spectateur, comme on pourrait l'attendre en ce lieu souvent réflexif d'un début de film, mais à une instance à la fois omniprésente et irreprésentable, désincarnée comme l'est la locutrice over. Avant et pendant cette incantation mystérieuse qui donne naissance au récit du film, l'entièreté du champ est occupée par la surface scintillante de l'eau sur laquelle se reflètent le bleu du ciel et le vert des cimes. A l'instant où la voix émerge pour énoncer son appel comme s'il s'agissait d'un rituel d'évocation, des gouttelettes de pluie commencent à s'abattre sur la surface aqueuse qu'elles animent par des mouvements concentriques, les reflets du paysage environnant devenant incertains, ondoyants comme une pensée vagabonde $\mathbf{2 1}$ (fig. 8).

Il n'est pas anodin que le film s'ouvre sur la rêverie d'une indigène: sa parole délimite l'ici de la terre dont les colons seront irrémédiablement étrangers, et annonce le ton d'un film qui "chante» une histoire plus qu'il ne la raconte. Associée à la terre, cette voix est pour l'instant sans origine, ou plutôt elle est l'origine. Un ici-maintenant sans cesse renouvelé à propos duquel le texte over brouillera parfois les repères, le spectateur étant plongé dans une représentation totalement subjective du temps. Le plan suivant nous montre Pocahontas (fig. 9) dans une forte contre-plongée qui ne nous permet pas de discerner son visage (et a fortiori ses lèvres, dont les mouvements nous feraient passer du 


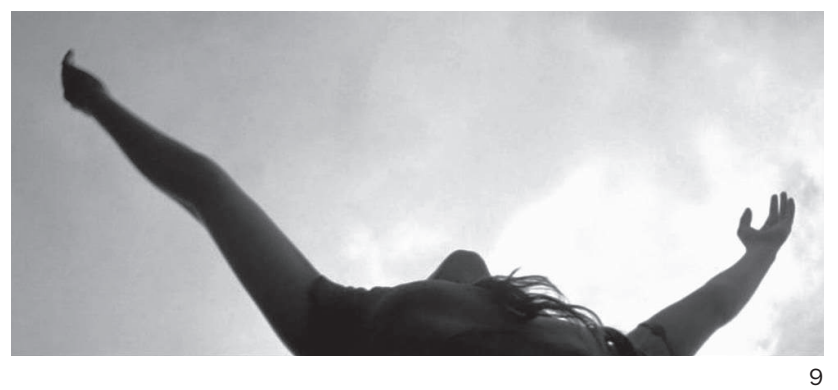

statut de off/over à celui de in): elle n'est pas un sujet parlant, ou du moins cette voix n'est pas encore la sienne. Car comment expliquer que Pocahontas s'exprime en anglais, alors que des séquences ultérieures sont dévolues à son apprentissage progressif de la langue des futurs colonisateurs? On pourrait faire l'hypothèse d'une narration rétrospective, mais cette dernière ne s'accorde nullement avec la forme impérative de la phrase. Il semble plus juste de considérer que, dans ces limbes où le monde du film s'apprête à s'instaurer, la glossolalie dont semble douée la narratrice est en fait la traduction, destinée au spectateur, d'une pensée qui n'a pas besoin du verbal pour entretenir un contact direct avec la nature. La terre primitive de Malick, domaine de l'esprit, est une utopie d'avant Babel. Dans le second plan de ce pré-générique, la jeune fille décrit d'ailleurs, les bras tendus, un mouvement de rotation qui s'inscrit de façon harmonieuse dans le prolongement des flots de l'image précédente. Or on apprendra plus tard, lorsqu'elle tentera de communiquer avec Smith, que ce geste est signe: il veut dire «ciel». En totale communion avec la nature dont elle imite physiquement les mouvements (notamment lorsqu'elle joue avec son frère), Pocahontas parle avec son corps. Par conséquent, la voix qui parvient aux oreilles du spectateur n’a pas de réalité phonique dans l'univers diégétique: jamais assujettie à une quelconque frontière, la voix-over, immatérielle, flottante, crée une continuité entre l'intériorité des personnages et les images du (nouveau) monde. Dans l'environnement édénique du début du film, la parole est une convention qui figure l'indicible. Cet usage de la voix-over l'identifie comme une manifestation infraverbale qui s'oppose à la langue parlée par les colons, représentée comme le produit d'une civilisation destructrice.

Des voix qui se dérobent au langage: la transparence, puis l'obstacle Lorsque John Smith, chargé de parlementer avec le roi indigène, part en mission, il exprime over la fascination que suscite en lui cette terre de 
22 Michael Henry, "Le Nouveau Monde. L'aurore désenchantée", Positif, n 540, février 2006, p. 14. liberté où semblent pouvoir se réaliser tous ses idéaux égalitaires. Son «monologue» n'est toutefois pas présenté sous la forme rationnelle d'une réflexion: les pensées de John semblent émaner de la nature elle-même qui "parle» en lui, ainsi qu'il l'insinue par certaines interrogations ("Qui es-tu, toi que j'entends vaguement?", ou "Quelle est cette voix qui parle en moi, qui me guide vers le meilleur?»). Pour l'audiospectateur du film, la voix-over de Farrell prend la place de cet appel intime dont il tente précisément d'invoquer la source, et qui se concrétisera temporairement dans l'attirance éprouvée pour Pocahontas, liée à la terre qu'elle prie constamment en l'appelant «Mère", alors qu'au combat, lors d'un soliloque qui suspend l'action, Smith se repent devant Dieu le Père de «ne pas avoir écouté sa Voix».

Il est surprenant de noter que le "monologue» over, généralement utilisé au cinéma pour exprimer un repli sur soi, fonctionne durant les séquences consacrées à la relation amoureuse entre Pocahontas et Smith comme un moyen de communication, le seul qui permette une certaine compréhension entre les partenaires, ainsi que le remarque Michael Henry, sensible à la dimension "musicale» des occurrences over dans Le Nouveau monde:

«Deux cœurs se mettent à nu, qui ne peuvent communiquer que par la parole intérieure. Ce n'est pas encore un dialogue, plutôt des monologues qui convergent peu à peu. Tels deux affluents qui se rejoignent en une même rivière. Au même titre que le paysage sonore, la voix off est une partition. »22

Henry souligne qu'il s'agit d'un phénomène progressif; il est vrai que l'intégralité de la première partie du film s'organise autour des changements qui touchent les modes de manifestation de la parole. Toutefois, dans l'univers désillusionné de Malick, la "convergence» n'est pas nécessairement un gage de bonheur. Au contraire : le passage de la communion des pensées over aux échanges verbaux in (ou off, puisque les voix diégétiques échappent le plus souvent au synchronisme vocolabial) sera un symptôme avant-coureur de la rupture entre les amants. L'évolution des relations dans le couple résulte donc du traitement même des voix-over et de leurs interactions. Au cœur de l'idylle, les amants s'expriment tour à tour over comme s'ils étaient unis dans un même espace spirituel, et, plus tard, la voix de Pocahontas demeurée chez les siens abolit la distance qui la sépare de son compagnon retourné au fort, flagellé pour avoir pris sa défense face aux colons qui projettent de la prendre en otage. Alors que seul Smith est montré en gros plan, elle s'adresse à lui dans une apostrophe qui débute significativement par l'injonction "Approche!» et se poursuit avec un ton qui connote 
l'intimité et la proximité. Lorsque les amants se retrouvent après les grands froids, un décalage s'est déjà instauré, manifeste dans l'entrelacs des voix masculine et féminine à travers les doutes formulés par Pocahontas, qui relativise l'idéalisme de son amant ${ }^{23}$. Au début du film, la voix-over permet de rendre perceptible l'infigurable de la transparence des consciences ${ }^{\mathbf{2 4}}$, mais ce pouvoir de l'intériorisation absolue s'amoindrit lorsque Pocahontas acquiert les rudiments de la langue anglaise, lorsqu'elle se familiarise avec des conventions qui extériorisent la signification, la coupant de son enracinement dans la terre au profit d'une socialisation ouverte à l'Autre. Le texte over tend alors à se rapprocher des paroles in associées à la tromperie, un travers inconnu des indigènes qui, selon les dires de Smith, sont «fidèles, exempts de toute fourberie». L'opposition entre ces types de manifestation vocale apparaît clairement lorsque Smith confesse en voix synchrone qu'il n'a "jamais vraiment été celui qu'elle croit». Pocahontas, d'abord totalement étrangère à l'idée de la dissimulation (contre laquelle elle devra ensuite lutter pour apprendre à aimer sincèrement son époux), se demande à cet instant même dans un murmure over: "Que dit-il ?». Cet entremêlement de voix in et over renvoie aux connotations qu'elles possèdent l'une et l'autre dans le système de valeurs mis en place par un film qui s'ouvre sur l'harmonie, puis fait place à une parole diégétique qui est le lieu d'une fracture.

\section{Retour aux sources}

L'intériorisation traditionnellement provoquée au cinéma par la voixover acquiert chez le personnage de Pocahontas des profondeurs qui excèdent la psychologie individuelle pour manifester l'enracinement ancestral des tribus indiennes de Virginie. Dans la conception panthéiste de Malick, le sujet parlant se dissout dans l'environnement: il n'est nulle part, c'est-à-dire partout. Le montage très elliptique des passages consacrés au couple souligne cette idée d'une absence d'ancrage spatial et temporel des personnages.

A l'instar de la musique, la voix-over est le véritable pouls du film, expression du vivant plus que celle d'un locuteur individué. En concevant prioritairement la voix comme une mélodie - dans La Ligne rouge surtout, les pauses qui interviennent dans les intermittences des voix constituent de véritables respirations qui ponctuent d'amples «mouvements»-, Malick renoue par d'autres biais avec des théoriciens du cinéma des années 1920 comme Louis Delluc ou Ricciotto Canudo, partisans du «musicalisme» et d'un rapport d'immédiateté entre l'œuvre et le spectateur ${ }^{25}$. L'ouïe est considérée dans ce contexte comme un mode perceptif archaïque qui favorise l'intégration de l'individu au
23 Les phrases suivantes se succèdent: (lui): "Lumière de ma vie"; (elle): "L'amour peut-il mentir?"; (lui): "Mon Amérique"; (elle): "Où es-tu, mon amour?".

24 Idéal rousseauiste abordé dans l'essai de Jean Starobinsky, Jean-Jacques Rousseau: la transparence et l'obstacle, Librairie Plon, Paris, 1958.

25 Voir à ce propos Laurent Guido, L'Age du Rythme. Cinéma, musicalité et culture du corps dans les théories françaises des années 19001930, Payot, Lausanne, 2007, pp. 231-236. 
26 Ce qu'un critique visiblement marqué par la conception musicaliste de l'époque formulait à propos des chants dans le film de Vidor pourrait s'appliquer aux voix over des deux dernières réalisations de Malick: "On ne sait si le chant naît de la lumière ou la lumière du chant. Ils paraissent s'engendrer mutuellement. II semble qu'on ne les perçoive plus par deux sens différents. La vue et l'ouïe n'existent plus. C'est un nouveau sens qui recueille et boit en même temps les harmonies sonores et visuelles" (Pour Vous, n 58, 28 décembre 1929, cité par Roger Icart, La Révolution du parlant, vue par la presse française, Institut Jean Vigo, Perpignan, 1988, p. 363). Mais alors qu'Hallelujah vise à représenter l'âme d'un peuple (le titre choisi pour l'exploitation française de ce film fut significativement L'Ame noire), Malick souligne, dans une même conception "animiste", l'absence d'harmonie entre les individus dont on épouse le point de vue (ce qu'implique en soi le contexte, qu'il s'agisse de la guerre ou de la colonisation).

27 Denis Vasse, L'Ombilic et la voix, Seuil, Paris, 1974; M. Chion, La Voix au cinéma, op. cit., chapitre 4. collectif, voire, chez Malick, au cosmos. Par contre, dans les histoires qu'il raconte, cette harmonie est vécue sur un mode déceptif: à l'exception des chants mélanésiens qui emplissent le paradis terrestre où s'est réfugié le déserteur Witt au début de La Ligne ronge (et qui reviennent à la fin du film, après la mort de ce soldat), la voix, cantonnée au repli sur soi du monologue intérieur, n'acquiert pas la dimension communautaire qu'elle possède dans un film comme Halleluyab (King Vidor, 1929), l'un des premiers talkies qui actualise sur le plan thématique l'idéal "musicaliste» de la fin du muet 26.

Grâce à ces voix susurrées qui attirent l'attention sur leur grain jusqu'à éclipser parfois le contenu des paroles, le film entretient avec le spectateur plongé dans l'obscurité de la salle de cinéma un rapport de proximité enveloppante, comme s'il s'agissait de retrouver la relation primitive qu'entretient le nourrisson avec l'espace intra-utérin. Dans le sillage des écrits du psychanalyste Denis Vasse, Michel Chion a souligné l'importance de la «Voix de la Mère» pour aborder les voix acousmatiques du cinéma ${ }^{27}$. Les invocations mélodieuses de "Mother» qui scandent Le Nouveau Monde s'inscrivent dans ce paradigme (tout en l'inversant), et se poursuivent lorsque Pocahontas elle-même donne la vie, instaurant dans le commentaire over une filiation entre sa procréation et l'œuvre de la nature, désormais domestiquée par le travail de son époux cultivateur. Dans La Ligne rouge, lorsque Witt utilise pour la première fois le pronom «je», c'est pour dire qu'il se souvient de sa mère mourante, amorçant l'un des rares passages véritablement narratifs du texte over. Les fugaces inserts mnésiques qui illustrent un peu plus tard ce souvenir - images à la composition systématiquement décentrée - confèrent une atmosphère onirique et renforcent l'effet de flottement résultant de l'ancrage indéterminé de la voix, cette dernière étant très momentanément identifiée comme synchrone. Qu'il s'agisse d'une mère décédée ou de la terre des ancêtres bientôt spoliée, les personnages tentent d'évoquer un passé originel fuyant que seule une voix désincarnée, elle-même située entre la présence et l'absence, semble susceptible de faire resurgir.

C'est le pouvoir mystique de la parole que Terrence Malick convoque dans ses deux derniers films, et qu'il décline dans ses diverses manifestations poétiques, immergeant le spectateur dans un maelström fascinant de sons et d'images, au même titre que ses personnages, plongés dans le milieu originel qui les accueille ou, d'une certaine manière, les retrouve. 


\section{ALAIN BOILLAT}

\section{DU BONIMENTEUR À LA VOIX-OVER}

\section{VOIX-ATTRACTION ET VOIX-NARRATION AU CINÉMA}

Du bonimenteur à la voix-over propose une réflexion historique et théorique sur la place et la fonction conférées à la voix, manifestation sonore foncièrement humaine, au sein du médium machinique qu'est le cinématographe.

En s'interrogeant d'abord sur l'accompagnement verbal qu'offrait un locuteur live (le «bonimenteur») à l'époque du cinéma des premiers temps, cet ouvrage rend compte des spécificités de l'oralité du cinéma parlé, puis confronte la médiation qui s'opère au sein de ce dispositif aux principes de la voix enregistrée, notamment en ce qui concerne le cas emblématique de la voix-over, étudiée ici dans ses implications narratologiques et énonciatives. Ainsi se dessinent deux grands axes qui permettent de renouveler la compréhension des phénomènes vocaux au cinéma en associant la prise en compte du texte (approche sémiologique) et du contexte (approche historique).

À travers l'examen de la voix-over et des discours qui lui ont été consacrés, l'auteur élabore un modèle inédit de l'énonciation au cinéma qui intègre la question de la matérialité de la voix et de son enregistrement. Par ailleurs, en abordant de façon systématique les modes de synchronisation et les relations sémantiques qui s'instaurent entre les mots et les images, il conçoit des instruments pour l'analyse filmique qu'il applique à certaines œuvres singulières du point de vue du régime vocal, telles que Le Roman d'un tricheur (Guitry), La Fiancée de Frankenstein (Whale), Lola Montès (Ophuls) ou Hiroshima mon amour (Resnais).

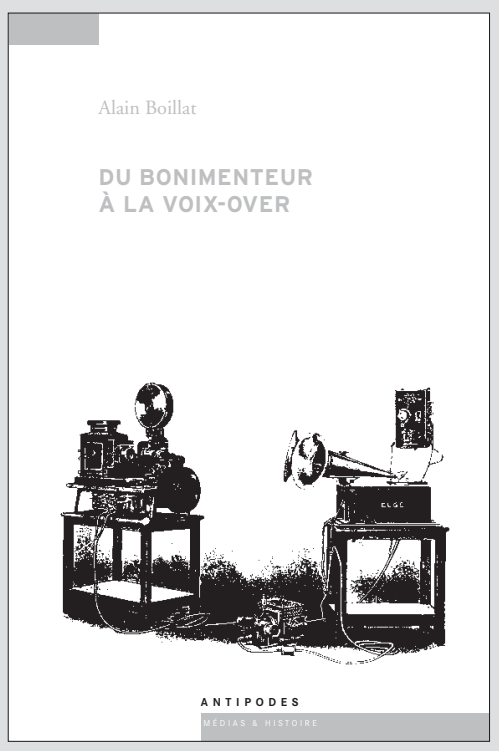

Éditions Antipodes Collection Médias et histoire 539 p., 978-2-940146-96-3, 50 francs suisses, 33 euros. www.antipodes.ch editions@antipodes.ch

Diffusion en Suisse: Zoé, Carouge.

Diffusion hors de Suisse: CID, Paris. 